\title{
Pharmacological effects of mitraphylline from Uncaria tomentosa in primary human monocytes: Skew toward M2 macrophages
}

S Montserrat-de la Paz ${ }^{1,2}, \mathrm{R}$ de la Puerta ${ }^{1}$, A Fernandez-Arche ${ }^{1}, \mathrm{~A} \mathrm{M} \mathrm{Quilez}^{1}$, F J G Muriana ${ }^{2}$, M D Garcia-Gimenez ${ }^{1}$, B Bermudez ${ }^{1, *}$

${ }^{1}$ Department of Pharmacology. School of Pharmacy. University of Seville, Spain

${ }^{2}$ Laboratory of Cellular and Molecular Nutrition. Instituto de la Grasa, CSIC, Seville, Spain

*Corresponding autor: Beatriz Bermudez. E-mail address: bbermudez@us.es

Department of Pharmacology, School of Pharmacy, Seville University

C/ Profesor Garcia Gonzalez 2, 41012, Seville, Spain.

Tel.: +34 954557443

Original Research Article

Running title: Anti-inflammatory effects of Mitraphylline

Funding Sources: None

\section{Authorship:}

The authors' responsibilities were as follows-MS, and BB: participated in the study design and performed the data analysis; MS, PR, FAA, QA, MFJG, GGM and BB: assisted with the editing of the manuscript; MS, PR, FAA, QA, MFJG, GGM and BB: acquired the data; and BB: wrote the first draft and finalized the manuscript. All authors participated in the analytic discussion of the results and approved the final version of the manuscript. 


\begin{abstract}
Ethnopharmacological relevance

Uncaria tomentosa (Willdenow ex Roemer \& Schultes) DC. (Rubiaceae) is a Peruvian thorny liana, commonly known as "cat's claw", and traditionally used in folk medicine to deal with several inflammatory diseases. Mitraphylline (MTP) is the most abundant pentacyclic oxindolic alkaloid (POA) from U.Tomentosa and has been reported to modify the inflammatory response. Herein, we have sought to identify the mechanisms underlying this modulatory effect of MTP on primary human monocytes and its ability to regulate differentiation processes on human primary monocyte and monocyte-derived macrophages.
\end{abstract}

\title{
Material and methods
}

In vitro studies with human primary monocytes and monocyte-derived macrophages were performed. Monocytes and M0 macrophages were exposed to MTP $(25 \mu \mathrm{M})$ and LPS (100 ng/mL). M0 macrophages were polarized to M1 and M2 phenotypes in the absence or presence of MTP. The activation state of monocytes/macrophages was assessed by flow cytometry, gene expression and protein analysis of different specific markers.

\section{Results}

In human primary monocytes, the incubation of MTP for $24 \mathrm{~h}$ reduced the number of classical (CD14++CD16-) and intermediated (CD14++CD16+) subsets when compared to untreated or LPS-treated cells. MTP also reduced the chemotactic capacity of human primary monocytes. In addition, MTP promoted the polarization of M0 macrophages toward an anti-inflammatory M2 phenotype, the abrogation of the release of pro-inflammatory cytokines such as TNF $\alpha$, IL6 or IL-1 $\beta$, as well as the restoration of markers for M2 macrophages in LPS-treated M1 macrophages. 


\section{Conclusions}

Our results suggest that MTP may be a key modulator for regulating the plasticity of monocytes/macrophages and the attenuation of the inflammatory response.

Chemical compounds studied in this article Mitraphylline (Pubchem CID: 94160)

KEYWORDS Uncaria tomentosa mitraphylline, monocytes, macrophages, polarization and inflammatory diseases.

ABBREVIATIONS MTP: mitraphylline, POA: pentacyclic oxindolic alkaloid, U. tomentosa: Uncaria tomentosa, LPS: lipopolysaccharide, TNFa: Tumor Necrosis Factor Alpha, IL: Interleukin, IFN $\gamma$ : Interferon gamma, CCR2: chemokine (C-C motif) receptor 2, CCL2: Chemokine (C-C motif) ligand 2, MCSF: Macrophage colony-stimulating factor. 


\section{Introduction}

Uncaria tomentosa (Willdenow ex Roemer and Schultes) DC and its relative Uncaria guanensis (Aublet) Gmell. (Rubiaceae) are plants widely used in folk and complementary medicine under the names of "Cat's claw". Uncarias are vines naturally growing in Peru and some other countries of South and Central America, and have there numerous applications in both human and animal medicine. U. tomentosa has been previously reported to deal with several inflammatory diseases (Müller et al., 2011; Rosenbaum et al., 2011), modulating the immune system and antitumor activities (Garcia-Gimenez., et al 2010; Rojas-Duran et al., 2012; Dietrich et al., 2014). Owing to the systematically growing applications of $U$. tomentosa in therapy, it is important to better recognize its pharmacological properties and safety of using.

Cat's claw contains more than 50 chemical constituents, including oxindole and indole alkaloids, polyphenols, among others (Heitzman et al., 2005). The majority alkaloids of Uncaria are indole and oxindole families (Laus et al., 2004), which are well recognized as phytochemical markers of this species with relevant pharmacological activities (Heitzman et al., 2005). Mitraphylline (MTP) is an oxindole alkaloid and the most ubiquitous alkaloid being present in 20 of 34 Uncaria species (Heitzman et al., 2005). Most of pharmacological studies have been focused on the fractions of either a plant species or a "crude drug", considered as a preparation from either a single or a mixture of Uncaria plants. However, fewer studies, if any, have paid attention to the bioactivity of isolated compound(s) such as MTP. Our research team have previously reported the isolation of MTP from the dried inner bark of U. tomentosa, the elucidation of its structure by NMR spectroscopy analysis and its antitumor properties on human sarcoma and breast cancer cells (García-Prado et al., 2007). However, little is known on the potential of MTP to mitigate the inflammatory response.

Monocytes are particularly involved in inflammatory processes (Shi et al., 2011). They are 
classified into three subsets: classical CD14++CD16-, intermediate CD14++CD16+, and non-classical CD14+CD16++ monocytes (Ziegler-Heitbrock et al., 2010). So far, classical monocytes represent the major fraction, about $85 \%$ of total monocytes that highly express the migratory chemokine receptor CCR2 (Ingersoll et al., 2010; Wong et al., 2011). They are professional phagocytes giving rise to classical M1 macrophages, which generate reactive oxygen species and secrete cytokines (TNF $\alpha$, IL-1 $\beta$, IL-6) in response to LPS during infection or inflammation (Mossig et al., 2009; Wong et al., 2011; Zwada et al., 2011). Intermediate monocytes display highest levels of CCR5, TLR4, CD163, and HLA-DR during activation and also secrete pro-inflammatory cytokines (Wong et al., 2011; Shantsila et al., 2011). Nonclassical monocytes are less granular and smaller in size, with lower expression of CCR2 than classical or intermediate subsets (Cross et al., 2010; Wong et al., 2011; Shantsila et al., 2011). These monocytes rich in CD16 are functionally involved in tissue repairing, patrolling, wound healing, and have the tendency to be polarized into non-classically M2 macrophages with an anti-inflammatory phenotype in response to a variety of stimuli, including IL-4 (Benoit et al., 2008; Gordon et al., 2010).

In the present study, we further investigated whether MTP may act as an immune-modulator and anti-inflammatory effector on human primary monocytes and monocyte-derived macrophages.

\section{Material and methods}

\subsection{Bark extract}

The plant material was collected in the Peruvian forest and was provided by Dr Carlos $\mathrm{S}$ González and was identified in the Botanic Department of the San Lorenzo Chemical Science School from the Asuncion University in Paraguay. The plant extract was obtained and the compound mitraphylline identified as previously described in (Garcia Prado et al., 2007). 
Briefly, $500 \mathrm{~g}$ of Uncaria tomentosa dried inner bark were treated with ammonium hydroxide and extracted with $500 \mathrm{ml}$ of dichloromethane for 3 times. After filtration, the obtained solution was concentrated in vacuo to afford a residue, which was dissolved in a hydrochloric acid solution (3\%). Ammonium hydroxide and dichloromethane were added again. After concentration in vacuo, the purified alkaloid fraction was obtained as a brown residue and the yield was $0.1 \%$.

\subsection{Isolation and identification of Mitraphylline}

The dried residue of alkaloid fraction $(0.5 \mathrm{~g})$ was subjected to a silica gel column chromatography, compacted with silica gel $0.063-0.2 \mathrm{~mm}(0.8 \times 25 \mathrm{~cm})$, followed by a gradient elution with various mixtures of n-hexane, dichloromethane, and methanol. Twentyfive fractions of about $15 \mathrm{~mL}$ each were collected. $200 \mathrm{mg}$ of white crystal (Mitraphylline) were obtained in the fractions $14-16$ (eluted with $\mathrm{CH} 2 \mathrm{Cl} 2 / \mathrm{MeOH}$ ) that corresponded to $87.2 \%$ of the total alkaloids. EIMS and $1 \mathrm{H}$ and $13 \mathrm{C}$ NMR experiments were carried out for its identification using an AVANCE 500 spectrophotometer. The following 2D NMR experiments COSY-DQF, (1H-13C)-HSQC, (1H-13C)-HMBC, and NOESY correlation were used to elucidate its structure. Furthermore, the $15 \mathrm{~N}$ chemical shift of the isomeric oxindole alkaloids, (1H-15N)-HMBC, was necessary to facilitate its characterization. The solvent used for NMR spectra was CDCl3 (Garcia Prado et al., 2007).

\subsection{Blood collection and monocyte isolation}

This study was conducted according to the guidelines of good clinical practice. Peripheral venous blood was isolated from healthy adult volunteers $(<35$ years old $)$ from the Hospital Virgen del Rocio at Seville. The investigation conformed to the principles outlined in the Helsinki Declaration of the World Medical Association. Donors declared that they were non- 
smokers and were not taking any medication. Blood samples were immediately collected into $\mathrm{K}_{3}$ EDTA-containing Vacutainer tubes (Becton Dickinson, NJ, USA) and peripheral blood mononuclear cells (PBMCs) were isolated by centrifugation over Ficoll Histopaque gradient (Sigma-Aldrich Chem, MO, USA). Monocytes were then isolated from PBMCs using positive selection with CD14 MicroBeads according to the manufacturer's instructions (MACS, Myltenyi Biotec, Madrid, Spain). Monocytes were tested for purity by CD14 fluorescein isothiocyanate labelling and fluorescence-activated cell sorter (FACS) analysis using a FACScanto II flow cytometer and FACSDiva software (Becton Dickinson Immunocytometry Systems, CA, USA) (Varela et al., 2011). Following isolation, the cells were suspended in a RPMI 1640 medium supplemented with L-glutamine, penicillin, streptomycin, and 1\% heatinactivated FCS. The monocytes were used within $24 \mathrm{~h}$ after isolation for the experiments.

\subsection{Monocyte differentiation and polarization into M1 and M2 macrophages}

Monocytes were induced to differentiate for 6 days in the presence of recombinant human MCSF (25 ng/mL) to obtain M0 macrophages. These cells were then cultured in RPMI 1640 supplemented with L-glutamine, penicillin, streptomycin, and 10\% heat-inactivated FCS. For M1 and M2 polarization, M0 macrophages were exposed to LPS (100 ng/mL) plus IFN $\gamma(20$ $\mathrm{ng} / \mathrm{mL})$ and to IL-4 (20 ng/mL), respectively, for additional $24 \mathrm{~h}$. MTP (25 $\mu \mathrm{M})$ was also added to the medium as indicated.

\subsection{Cell viability}

Monocytes and macrophages seeded in 96-well plates $\left(1 \times 10^{5}\right.$ cells/well) were incubated in the presence (or absence) of different MTP concentrations for $24 \mathrm{~h}$. At the end of the exposure time, cell viability was analysed by Annexin $\mathrm{V}$ binding and using a FACScanto II flow cytometer with a CellQuest software (BD). 


\subsection{Nitrite and cytokine production}

Cells in 24-well plates were treated (or untreated) with MTP $(25 \mu \mathrm{M})$, and $30 \mathrm{~min}$ later stimulated with LPS $(100 \mathrm{ng} / \mathrm{mL})$ for $24 \mathrm{~h}$. The culture supernatants $(100 \mu \mathrm{L})$ were transferred to a 96-well assay plate mixed with Griess reagent (Sigma-Aldrich Chem) and incubated for $15 \mathrm{~min}$ at room temperature. The amount of nitrite, as an index of NO generation (Csonka et al 2014), was determined by the absorbance at $540 \mathrm{~nm}$ in an ELISA reader (BioTek, Bad Friedrichshall, Germany). After the extrapolation from a standard curve with sodium nitrite, the results were expressed as the percentage of nitrite compared with that of cells treated with only LPS. Dexamethasone $(1 \mu \mathrm{M}$, Sigma-Aldrich Chem) was used as positive control.

The cytokines levels of IL-1 $\beta$, IL- 6 , and TNF $\alpha$, which were released into cell supernatants, were measured by ELISA, following the indications of the manufacturer (Diaclone).

\subsection{Immunostaining analysis by FACS}

Membrane surface expression of CD16 (PE anti-human CD16, Miltenyi Biotec), CD14 (APC-Cy7 anti-human CD14, Miltenyi Biotec), and CCR2 (APC anti-human CCR2, BD) in monocytes was assessed by FACS. According to the manufacturer's instructions, $5 \times 10^{5}$ of purified monocytes, after in vitro stimulation with (or without) LPS (100 ng/mL), were incubated with the above antibodies in the dark, at room temperature for $15 \mathrm{~min}$. Thereafter, cells were fixed and erythrocytes were lysed with a volume $(20 \times)$ of FACS lysing solution (BD). Fluorescence intensity was measured in a FACSCanto II flow cytometer with a CellQuest software (BD). Results were analysed using the Win-List software package (Verity Software House, ME, USA). Mean fluorescence intensity (MFI) of $10^{4}$ counted cells was measured in each sample. Monocytes were gated as forward scatter ${ }^{\text {high }}$ (FSC ${ }^{\text {high }}$ )-side 
scatter $^{\text {high }}\left(\mathrm{SSC}^{\text {high }}\right)$ cells. Expression levels were presented as MFI corrected for nonspecific binding of isotype control antibodies.

\subsection{RNA isolation and $q R T-P C R$ analysis}

Total RNA was extracted from cells by using Trisure Reagent (Bioline), as instructed by the manufacturer. RNA quality was assessed by $\mathrm{A}_{260} / \mathrm{A}_{280}$ ratio in a NanoDrop ND-1000 Spectrophotometer (Thermo Scientific). RNA (1 $\mu \mathrm{g})$ was subjected to reverse transcription (iScript, Bio-Rad, CA, USA) according to the manufacturers' protocol. An amount of $20 \mathrm{ng}$ of the resulting cDNA was used as a template for real-time PCR amplifications. The mRNA levels for specific genes were determined in a MX3000P system (Stratagene). For each PCR reaction, cDNA template was added to Brilliant SYBR green QPCR Supermix (Bio-Rad) containing the primer pairs for either gene or for glyceraldehyde 3-phosphate dehydrogenase (GAPDH) and hypoxanthine phosphoribosyltransferase ( $\beta$-actine) as housekeeping genes (Table 1). All amplification reactions were performed in triplicate and average threshold cycle (Ct) numbers of the triplicates were used to calculate the relative mRNA expression of candidate genes. The magnitude of change of mRNA expression for candidate genes was calculated by using the standard $2^{-(\Delta \Delta \mathrm{Ct})}$ method. All data were normalized to endogenous reference (GAPDH and $\beta$-actine) gene content and expressed as percentage of controls.

\subsection{Immunoblotting detection}

Macrophages derived from primary monocytes $\left(1 \times 10^{6}\right.$ cells $\left./ \mathrm{mL}\right)$ were treated (or untreated) with MTP $(25 \mu \mathrm{M})$ and LPS $(100 \mathrm{ng} / \mathrm{mL})$ for $18 \mathrm{~h}$. After incubation, cells were rinsed, scraped off, and collected in ice-cold PBS containing a cocktail of protease and phosphatase inhibitors and processed to isolate cytoplasmic proteins. Protein concentration was measured for each sample using a protein assay reagent (Bio-Rad) according to the Bradford's method 
and using $\gamma$-globulin as a standard (Bradford, 1976). Aliquots of supernatant containing equal amount of protein $(20 \mu \mathrm{g})$ were separated on $10 \%$ acrylamide gel by SDS polyacrylamide gel electrophoresis. In the next step, the proteins were electrophoretically transferred into a nitrocellulose membrane and incubated with specific primary antibodies, polyclonal mouse anti-human TNF $\alpha$ and IL1- $\beta$ (Abcam, London, UK) and monoclonal mouse anti-human bactin (Sigma, Milan, Italy), overnight at $4{ }^{\circ} \mathrm{C}$. After rinsing, the membranes were incubated with a horseradish peroxidase-labelled (HRP) secondary antibody anti-rabbit (Cayman Chemical, MI, USA) (1:50000) or anti-mouse (Dako, GA, USA) (1:2000) containing blocking solution for $1-2 \mathrm{~h}$ at room temperature. To prove equal loading, the blots were analysed for $\beta$-actin expression using an anti- $\beta$-actin antibody (Sigma Aldrich Chem). Immunodetection was performed using enhanced chemiluminescence light-detecting kit (Pierce, IL, USA). The immunosignals were captured using a LAS-3000 Imaging System from Fujifilm Image Reader (Stamford, USA). Following normalization to the housekeeping loading control, the signals were analysed and quantified by an Image Processing and Analysis in Java (Image J, Softonic).

\subsection{Wound healing assay}

M0/M1 macrophages were plated in a 24-well tissue culture plate in complete growth medium to attain $\sim 80 \%$ confluence. Cells were allowed to adhere for $24 \mathrm{~h}$ and then a line was scraped with a P-1000 micropipette tip. Non-adherent cells were aspirated away, and complete growth media was replaced. Macrophages were then incubated with MTP in the presence (or absence) of $10 \mathrm{nM}$ recombinant human MCP-1 (PeproTech EC, London, UK). Serial images were taken at designated time points at $\times 100$ magnification with Image $\mathrm{J}$ software, a standardized grid was placed over the images across the breadth of the denuded 
zone, and cells migrating into this zone were counted in a blinded fashion. Each quantified sample represents counts from four different wells with two serial images taken per well.

\subsection{Data analysis}

All values in the figures and text are expressed as arithmetic means \pm standard error (SEM). Data were evaluated with Graph Pad Prism Version 5.01 software (CA, USA). The statistical significance of any difference in each parameter among the groups was evaluated by one-way analysis of variance (ANOVA) following Dunnett's multiple comparisons test as post hoc test. $\mathrm{P}$ values less than 0.05 were considered statistically significant.

\section{Results}

\subsection{MTP upsetting the balance of primary monocyte subsets}

Monocytes were analysed for cell viability and different subset markers after $24 \mathrm{~h}$ of incubation with MTP $(25 \mu \mathrm{M})$. Contrarily to staurosporine $(1 \mu \mathrm{M})$ that was used as a positive control of cell death and caused spontaneous apoptosis $(>52 \%)$ and necrosis $(>19 \%)$, MTP did not induce any significant loss of viability in monocytes (Fig. 1A). Next, we measured surface expression of phenotypic markers (CD14 and CD16) to distinguish between classical, intermediate, and non-classical monocyte subsets. To approach this, primary monocytes were exposed to LPS (100 ng/mL) alone or in the presence of MTP. Compared to control untreated cells, LPS significantly increased the expression of CD14 in the classical and intermediate monocyte subsets (Fig. 1B). MTP did not only abrogate this LPS-related effect by decreasing the expression of CD14, but also by significantly increasing the expression of CD16 and thereby of non-classical monocyte subsets. In addition, MTP significantly decreased CCR2 expression in the classical monocyte subset (Fig. 1C). 


\subsection{MTP shifts M1 to M2 macrophage phenotype}

Monocytes were cultured for 6 days with human recombinant M-CSF ( $25 \mathrm{ng} / \mathrm{mL})$ to mature into M0 macrophages. The FACS analysis showed that 97\% of M0 cells were positive for the macrophage marker CD68 (Fig. 2A). These cells were then treated with IFN $\gamma(20 \mathrm{ng} / \mathrm{mL})$ or IL-4 (20 ng/mL) for $24 \mathrm{~h}$ to generate M1 or M2 macrophages, respectively. Gene expression of phenotypic markers for M1 (CD64, CD80, and CD163) and M2 (CD200r and MR) macrophages was examined. As expected, CD64, CD80, and CD163 were upregulated by LPS plus IFN $\gamma$ in M1 macrophages (Fig. 2B-D), whereas CD200r and MR were upregulated by IL-4 in M2 macrophages (Fig. 2E-F). The presence of MTP during the treatment of M0 macrophages with IFN $\gamma$ prevented the expression of M1 markers, reaching comparable levels to those found in M2 macrophages, and increased M2 markers.

Based on active aerobic glycolytic program in M1 macrophages that results in high production of nitrite oxides (NO2-) (Galván-Peña et al., 2014), we tested the influence of MTP on NO2- production in M0 macrophages treated with IFN $\gamma$. It was interesting to note that MTP completely abolished to normalize NO levels in these cells (Fig. 2G).

\subsection{MTP restricts CCR2-dependent migration}

Classically activated M1 macrophages are recruited into inflamed tissues partly via CCR2 (Fadini et al., 2014) and the CCR2-CCL2 axis is one of the key regulatory pathways in macrophage migration (Izumi et al., 2013). In contrast, alternatively activated M2 macrophages are characterized by their poor migratory capacity and key role in the resolution of the inflammatory response and regenerative repair in wound healing. Compatible with this notion, we found increased gene expression of CCR2 and CCL2 in M1 macrophages but decreased gene expression of CCR2 and CCL2 in M2 macrophages when compared to M0 macrophages (Fig. 3A-B). Of note, MTP significantly decreased gene expression of CCR2 in 
M0 macrophages treated with LPS and IFN $\gamma$ to levels close to those observed in M2 macrophages (Fig. 3A). These findings were in agreement with an MTP-induced reduction of cell motility during the treatment of M0 macrophages to be polarized into M1 macrophages, even when CCL2 was added (Fig. 3C).

\subsection{MTP prevents secretion of pro-inflammatory cytokines}

Next, we investigated the effect of MTP on macrophage activation by determining its ability to modulate pro-inflammatory cytokine production in response to LPS. M0 macrophages were exposed to LPS in the absence or presence of MTP for $24 \mathrm{~h}$. MTP induced a decrease in the gene expression (Fig. 4A-C), and the protein expression (Fig. 4D-E) and secretion (Fig. 4F-H) of TNF $\alpha$, IL-1 $\beta$, and IL-6 in LPS-treated M0 macrophages.

\section{Discussion}

The uses of western therapies to treat inflammatory diseases are often suboptimal in terms of their associated side effects and cost. Currently, U. tomentosa is known as an herbal drug in several South American countries due to its beneficial effects on DNA repair and immune function (Sheng et al., 2000; Faria et al., 2012). Uncaria has proven to be a very valuable genus to the discovery and utilization of medicinal natural products that includes alkaloids. Therefore, the potential for development of leads from Uncaria needs to grow, particularly in the area of immunology, inflammation, and vascular-related disorders. The immunostimulant activity of pentacyclic oxindolic alkaloids (POA) from $U$. tomentosa may occur by increasing phagocytosis of granulocytes and macrophages, and by blocking the proliferation of myeloid cells (Keplinger et al., 1999). MTP is the most ubiquitous and abundant POA from $U$. tomentosa, and a recognised molecule with potent immunomodulatory and anti-inflammatory effects (Domingues et al., 2011). However, little is know about the mechanisms through 
which those POA, and specifically MTP, are acting on the immune system and inflammatory pathways.

One of the pivotal cells for the initiation of inflammation are monocytes, which exhibit different phenotypes according to the expression of the surface markers CD14 and CD16 (Zawada et al., 2012). CD14 responses to bacterial LPS via toll-like receptor 4 (Hansson et al., 2011), whereas CD16 (Fc $\gamma \mathrm{R}-\mathrm{III})$ renders monocytes targets for activation by IgGcontaining immune complexes (IC) (Grivennikov et al., 2011). The majority of circulating monocytes belong to the classical monocytes $\mathrm{CD} 14^{++} \mathrm{CD} 16^{-}$; the remaining monocytes are described as the intermediate $\mathrm{CD} 14^{++} \mathrm{CD} 16^{+}$and the non-classical $\mathrm{CD} 14^{+} \mathrm{CD} 16^{++}$subsets (Zawada et al., 2011). The intermediate monocyte subset has a pro-inflammatory phenotype that actively produces TNF $\alpha$ (in response to LPS), IL-1 $\beta$, IL-6, and leads to the progression of numerous inflammatory disorders such as atherosclerosis (Rogacev et al., 2014). It is necessary to maintain the proper balance among the different monocytes subsets for the appropriated inflammatory state. This study is the first to demonstrate that MTP may regulate the CD14/CD16 signature in human monocytes. MTP counteracted the imbalance of increased classical $\left(\mathrm{CD} 14^{++} \mathrm{CD} 16^{-}\right)$and intermediate $\left(\mathrm{CD} 14^{++} \mathrm{CD} 16^{+}\right)$monocytes, and decreased non-classical $\left(\mathrm{CD} 14^{+} \mathrm{CD} 16^{++}\right)$monocytes that induced LPS. This ability of MTP involved a decrease of CD14 expression and an increase of CD16 expression, resulting in a population of monocytes with lower classical and higher non-classical subsets. We also found that LPS-treated monocytes exposed to MTP were less migratory. Despite of the fact that CCR2 was less expressed in intermediate and non-classical monocytes in comparison with classical monocytes, MTP was shown to diminish CCR2 surface expression in all monocyte population but particularly in classical monocytes when co-treated with LPS. These findings could be explained due to the fact that CCR2 is not a static entity but is subjected to dynamic regulation in a time-dependent fashion by LPS (Heesen et al., 2006). 
In response to inflammatory stimuli (i.e., LPS), the classical monocytes are selectively trafficked, in first instance, to the sites of inflammation, producing pro-inflammatory cytokines and contributing to local and systemic inflammation (Yang et al., 2014). These monocytes are highly infiltrative and can be differentiated into inflammatory macrophages, M1 acting in the acute phase of inflammation. In steady state, the non-classical patrolling monocytes patrol the vascular endothelium and become resident macrophages when they infiltrate into the inflamed tissue. During the late phase of inflammation, differentiated nonclassical monocytes (formally M2 macrophages) acquire an anti-inflammatory phenotype, which contribute to a shutdown of activated leukocytes and repair of damaged tissues (Murray et al., 2011). Sustained inflammation degenerates in chronic inflammatory diseases (Hotamisligil., 2006). Therefore, we decided to evaluate whether the shift from classical toward non-classical monocytes caused by MTP could lead to a different behaviour during polarization of macrophages derived from primary monocytes (M0 macrophages). It was exciting to observe that these macrophages, when treated with MTP, were hyporeactive to inducers (LPS and IFN $\gamma$ ) of M1 polarization and displayed a strong alternative activation as similarly occurred in response to the inducer (IL-4) of M2 polarization. Moreover, we found that M1 macrophages underwent a phenotypic regression toward M2 macrophages in the presence of MTP. These findings suggesting that MTP not only might have a potential role to reduce the M1/M2 macrophage ratio by an increased availability of non-classical monocytes, but also by interfering the polarization of M1 macrophages in a manner that is reminiscent of macrophage polarization to M2. In accordance with this notion, MTP dramatically reduced CCR2 expression and abolished the chemotactic capacity of macrophages during M1 polarization. MTP also decreased the expression and secretion of several pro-inflammatory cytokines, which agreed with a previous study reporting, decreased plasma levels of TNF $\alpha$, IL- $1 \alpha$, and IL-1 $\beta$ by oral administration of MTP in a murine model of LPS-induced 
inflammation (Rojas-Duran et al., 2012). The additional effect of MTP to abolish nitric oxide production during macrophage polarization to M1 expands the current knowledge on antioxidant effects of other chemicals from Uncaria such as tannins and condensed tannins (Desmarchelier et al., 1997). Collectively, we showed for the first time that MTP, one of the major alkaloids of $U$. tomentosa, is able to immuno-modulate the phenotype and function of innate immune cells that are intimately involved in inflammation. Our results indicate unknown qualities of MTP, which are partly responsible for the anti-inflammatory activity of Uncaria's extracts. These properties arise from its potency to switch pro-inflammatory state through immune system modulation, precisely, by skewing human monocytes to less inflammatory populations. Indeed, Uncaria's extracts have been already shown to inhibit antiinflammatory responses via nuclear factor-kB $(\mathrm{NF}-\mathrm{kB})$, which regulates proinflamatory cytokines secretion (Aguilar et al.,2002). However, anti-inflamtory Uncaria's activity is not solely due to MTP, certainly, the presence of this major alkaloid provides many of the antiinflammatory properties conferred on U. tomentosa.

\section{Conclusion}

In conclusion, we are the first to show that MTP might confer protection against inflammatory processes by skewing macrophage polarization to M2. This natural compound from $U$. tomentosa could be considered as a new player for therapy against inflammatory disorders. Nevertheless, further investigation must be conducted to approach this new therapeutic horizon.

\section{Acknowledgements}

This work was supported by the University of Seville, "V Own Research Plan" contract to BB and QA. MS has the benefit of a FPI fellowship (BES-2012-056104) of MICINN. 


\section{Conflicts of interest}

We declare that we do not have any financial or other relationships that might lead to a conflict of interest.

\section{Reference}

Aguilar, J.L., Rojas, P., Marcelo, A., Plaza, A., Bauer, R., Reininger, E., Klaas, C.A., Merfort, I., 2002. Anti-inflammatory activity of two different extracts of Uncaria tomentosa (Rubiaceae). J. Ethnopharmacol. 81, 271-/276.

Allen-Hall, L., Arnason, J.T., Cano, P., Lafrenie, R.M., 2009. Uncaria tomentosa acts as a potent TNF-alpha inhibitor through NF-kappaB. J. Ethnopharmacol. 127(3), 685-693.

Benoit, M., Desnues, B., Mege, J.L., 2008. Macrophage polarization in bacterial infections. J Immunol. 181, 3733-3739.

Cros. J., Cagnard, N., Woollard, K., Patey, N., Zhang, S.Y., Senechal, B., Puel, A., Biswas, S.K., Moshous, D., Picard, C., Jais, J.P., D'Cruz, D., Casanova, J.L., Trouillet, C., Geissmann, F., 2010. Human CD14dim monocytes patrol and sense nucleic acids and viruses via TLR7 and TLR8 receptors. Immunity. 33, 375-386.

Csonka, C., Páli, T., Bencsik, P., Görbe, A., Ferdinandy, P., Csont, T., 2015. Measurement of nitric oxide in biological samples. Br J Pharmacol. Br. J. Pharmacol. 172(6), 1620-1632.

Dietrich, F., Kaiser, S., Rockenbach, L., Figueiró, F., Bergamin, L.S., da Cunha, F.M., Morrone, F.B., Ortega, G.G., Battastini, A.M., 2014. Quinovic acid glycosides purified fraction from Uncaria tomentosa induces cell death by apoptosis in the T24 human bladder cancer cell line. Food. Chem. Toxicol. 67, 222-229. 
Domingues, A., Sartori, A., Valente, L.M., Golim, M.A., Siani, A.C., Viero, R.M., 2011. Uncaria tomentosa aqueous-ethanol extract triggers an immunomodulation toward a Th2 cytokine profile. Phytother. Res. 25(8), 1229-1235.

Fadini, G.P., Simoni, F., Cappellari, R., Vitturi, N., Galasso, S., Vigili de Kreutzenberg, S., Previato, L., Avogaro, A., 2014. Pro-inflammatory monocyte-macrophage polarization imbalance in human hypercholesterolemia and atherosclerosis. Atherosclerosis. 237(2), 805808.

Farias, I.L., Araújo, M.C., Farias, J.G., Rossato, L.V., Elsenbach, L.I., Dalmora, S.L., Flores, N.M., Durigon, M., Cruz, I.B., Morsch, V.M., Schetinger, M.R., 2012. Uncaria tomentosa for Reducing Side Effects Caused by Chemotherapy in CRC Patients: Clinical Trial. Evid. Based. Complement. Alternat. Med. 2012:892182.

García-Gimenez, M.D., Garcia-Prado, E., Saenz-Rodríguez, T., Fernandez-Arche, A., De la Puerta, R., 2010. Cytotoxic effect of the pentacyclic oxindole alkaloid mitraphylline isolated from Uncaria tomentosa bark on human Ewing's sarcoma and breast cancer cell lines. Planta. Med. 76(2): 133-136.

García-Prado, E., García-Gimenez, M.D., De la Puerta-Vázquez, R., Espartero-Sánchez, J.L., Sáenz-Rodríguez, M.T., 2007. Antiproliferative effects of mitraphylline, a pentacyclic oxindole alkaloid of Uncaria tomentosa on human glioma and neuroblastoma cell lines. Phytomedicine. 14(4), 280-284.

Gordon, S., Martinez, F.O., 2010. Alternative activation of macrophages: mechanism and functions. Immunity. 32, 593-604.

Gordon, S., Taylor, P.R., 2005. Monocyte and macrophage heterogeneity. Nat. Rev. Immunol. 5, 953-964. 
Grivennikov, S.I., Karin, M., 2011. Inflammatory cytokines in cancer: tumour necrosis factor and interleukin 6 take the stage. Ann. Rheum. Dis. 70 Suppl 1:i104-8.

Hansson, G.K., Hermansson, A., 2011. The immune system in atherosclerosis. Nat. Immunol 2: $204-212$.

Heesen, M., Renckens, R., de Vos, A.F., Kunz, D., van der Poll, T., 2006. Human endotoxemia induces down-regulation of monocyte $\mathrm{CC}$ chemokine receptor 2. Clin. Vaccine. Immunol. 13(1), 156-159.

Heitzman, M.E., Neto, C.C., Winiarz, E., Vaisberg, A.J., Hammond, G.B., 2005. Ethnobotany, phytochemistry and pharmacology of Uncaria (Rubiaceae). Phytochemistry. 66, $5-29$.

Hotamisligil, G.S., 2006. Inflammation and metabolic disorders . Nature. 444, 860-867.

Ingersoll, M.A., Spanbroek, R., Lottaz, C., Gautier, E.L., Frankenberger, M., Hoffmann, R., 2010. Comparison of gene expression profiles between human and mouse monocyte subsets. Blood. 115(3), e10-9.

Izumi, K., Fang, L.Y., Mizokami, A., Namiki, M., Li, L., Lin, W.J. Chang, C., 2013. Targeting the androgen receptor with siRNA promotes prostate cancer metastasis through enhanced macrophage recruitment via CCL2/CCR2-induced STAT3 activation. EMBO. Mol. Med. 5(9), 1383-1401.

Keplinger, K., Laus, G., Wurm, M., Dierich, M.P., Teppner, H., 1999. Uncaria tomentosa (Willd.) DC.--ethnomedicinal use and new pharmacological, toxicological and botanical results. Ethnopharmacol. 64(1), 23-34.

Laus, G., 2004. Advances in chemistry and bioactivity of the genus Uncaria. Phytother. Res. 
18(4), 259-274.

Mantovani, A., Sica, A., Locati, M., 2005. Macrophage polarization comes of age. Immunity $23,344-346$.

Müller, A.C., Kanfer, I., 2011. Potential pharmacokinetic interactions between antiretrovirals and medicinal plants used as complementary and African traditional medicines. Biopharm. Drug. Dispos. 32(8), 458-470.

Murray, P.J., Wynn, T.A., 2011. Protective and pathogenic functions of macrophage subsets. Nat. Rev. Immunol. 11(11), 723-737.

Rogacev, K.S., Zawada, A.M., Emrich, I., Seiler, S., Böhm, M., Fliser, D., Woollard, K.J., Heine, G.H., 2014. Lower Apo A-I and lower HDL-C levels are associated with higher intermediate CD14++CD16+ monocyte counts that predict cardiovascular events in chronic kidney disease. Arterioscler. Thromb. Vasc. Biol. 34(9), 2120-2127.

Rojas-Duran, R., González-Aspajo, G., Ruiz-Martel, C., Bourdy, G., Doroteo-Ortega, V.H., Alban-Castillo, J., Robert, G., Auberger, P., Deharo, E., 2012. Anti-inflammatory activity of Mitraphylline isolated from Uncaria tomentosa bark. J. Ethnopharmacol. 143, 801-804.

Rosenbaum, C.C., O'Mathúna, D.P., Chavez, M., Shields, K., 2010. Antioxidants and antiinflammatory dietary supplements for osteoarthritis and rheumatoid arthritis. Altern. Ther. Health. Med. 16(2), 32-40.

Shantsila, E., Wrigley, B., Tapp, L., Apostolakis, S., Montoro-Garcia, S., Drayson, M.T., Lip, G.Y., 2011. Immunophenotypic characterization of human monocyte subsets: possible implications for cardiovascular disease pathophysiology. J. Thromb. Haemost. 9(5), 10561066 
Sheng, Y., Pero, R.W., Wagner. H., 2000. Treatment of chemotherapy-induced leukopenia in a rat model with aqueous extract from Uncaria tomentosa. Phytomedicine. 7(2), 137-143.

Shi, C., Eric, G., Pamer, E.G., 2011. Monocyte recruitment during infection and inflammation. Nature Reviews Immunology. 11, 762-774.

Valera, L.M., Ortega, A., Bermudez, B., Lopez, S., Pacheco, Y.M., Villar, J., Abia, R., Muriana, F.J., 2011. A high-fat meal promotes lipid-load and apolipoprotein B-48 receptor transcriptional activity in circulating monocytes. Am. J. Clin. Nutr. 93, 918-925.

Wong, K.L., Yeap, W.H., Tai, J.J., Ong, S.M., Dang, T.M., Wong, S.C., 2012. The three human monocyte subsets: implications for health and disease. Immunol. Res. 53(1-3), 41-57.

Yang, J., Zhang, L., Caijia, Y., Yang, X.F., Wang, H., 2014. Monocyte and macrophage differentiation: circulation inflammatory monocyte as biomarker for inflammatory diseases. Biomarker Research. 2,1.

Zawada, A.M., Rogacev, K.S., Rotter, B., Winter, P., Marell, R.R., Fliser, D., Heine GH., 2011. SuperSAGE evidence for CD14++CD16+ monocytes as a third monocyte subset. Blood. 118(12), e50-61.

Zawada, A.M., Rogacev, K.S., Schirmer, S.H., Sester, M., Böhm, M., Fliser, D., Heine, G.H., 2012. Monocyte heterogeneity in human cardiovascular disease. Immunobiology. 217(12), 1273-1284.

Ziegler-Heitbrock, L., Ancuta, P., Crowe, S., Dalod, M., Grau, V., Hart, D.N., Leenen, P.J., Liu, Y.J., MacPherson, G., Randolph, G.J., Scherberich, J., Schmitz, J., Shortman, K., Sozzani, S., Strobl, H., Zembala, M., Austyn, J.M., Lutz, M.B., 2010. Nomenclature of monocytes and dendritic cells in blood. Blood. 116(16), e74-80. 
Table 1. Sequences of qRT-PCR primers for gene expression analysis.

\begin{tabular}{|c|c|c|c|}
\hline Target & $\begin{array}{l}\text { GenBank } \\
\text { accession number }\end{array}$ & Direction & Sequence $\left(5^{\prime} \rightarrow 3^{\prime}\right)$ \\
\hline CD80 & NM_005191.3 & $\begin{array}{l}\text { Forward } \\
\text { Reverse }\end{array}$ & $\begin{array}{l}\text { GGGAAAGTGTACGCCCTGTA } \\
\text { GCTACTTCTGTGCCCACCAT }\end{array}$ \\
\hline CD200R & NM_138940.2 & $\begin{array}{l}\text { Forward } \\
\text { Reverse }\end{array}$ & $\begin{array}{l}\text { GTTGCCCTCCTATCGCATTA } \\
\text { TGGAAATTCCCATCAGGTGT }\end{array}$ \\
\hline MR & NM_002438.3 & $\begin{array}{l}\text { Forward } \\
\text { Reverse }\end{array}$ & $\begin{array}{l}\text { GGCGGTGACCTCACAAGTAT } \\
\text { ACGAAGCCATTTGGTAAACG }\end{array}$ \\
\hline CD64 & NM_000566.3 & $\begin{array}{l}\text { Forward } \\
\text { Reverse }\end{array}$ & $\begin{array}{l}\text { GTCCAAATCTCCAAGTGCGG } \\
\text { CCCAAGTATGAGAGCAGCGT }\end{array}$ \\
\hline CD163 & NM_203416.3 & $\begin{array}{l}\text { Forward } \\
\text { Reverse }\end{array}$ & $\begin{array}{l}\text { TTGCCAGCAGCTTAAATGTG } \\
\text { AGGACAGTGTTTGGGACTGG }\end{array}$ \\
\hline IL-1 $\beta$ & NM_000576 & $\begin{array}{l}\text { Forward } \\
\text { Reverse }\end{array}$ & $\begin{array}{l}\text { GGGCCTCAAGGAAAAGAATC } \\
\text { TTCTGCTTGAGAGGTGCTGA }\end{array}$ \\
\hline TNF $\alpha$ & NM_000594 & $\begin{array}{l}\text { Forward } \\
\text { Reverse }\end{array}$ & $\begin{array}{l}\text { TCCTTCAGACACCCTCAACC } \\
\text { AGGCCCCAGTTTGAATTCTT }\end{array}$ \\
\hline IL-6 & NM_000600 & $\begin{array}{l}\text { Forward } \\
\text { Reverse }\end{array}$ & $\begin{array}{l}\text { TACCCCCAGGAGAAGATTCC } \\
\text { TTTTCTGCCAGTGCCTCTTT }\end{array}$ \\
\hline IL-10 & NM_000572 & $\begin{array}{l}\text { Forward } \\
\text { Reverse }\end{array}$ & $\begin{array}{l}\text { GCCTAACATGCTTCGAGATC } \\
\text { TGATGTCTGGGTCTTGGTTC }\end{array}$ \\
\hline CCR2 & NM_001123396.1 & $\begin{array}{l}\text { Forward } \\
\text { Reverse }\end{array}$ & $\begin{array}{l}\text { TGCCTGACTCACACTCAAGG } \\
\text { GGCTTCTCAGCAACTGAACC }\end{array}$ \\
\hline MCP-1 & NM_002982.3 & Forward & CCCCAGTCACCTGCTGTTAT \\
\hline
\end{tabular}




\begin{tabular}{|l|l|l|l|}
\hline & & Reverse & ACGAAGCCATTTGGTAAACG \\
\hline GAPDH & NM_001289746 & Forward & AACATGGCCTCCAAGGAGTA \\
\hline$\beta$-Actin & NM_001101 & Reverse & CCAGCAGTGAGGGTCTCTCT \\
\hline
\end{tabular}




\section{FIGURES LEGENDS}

Figure 1. (A) Apoptosis and necrosis representative pictures of flow cytometry analysis from human monocytes isolated from PBMCs (primary monocytes) after $24 \mathrm{~h}$ incubation with MTP $(25 \mu \mathrm{M})$ or Staurosporine $(1 \mu \mathrm{M})$. (B) FACS analysis of monocyte surface markers CD14 and CD16 after $24 \mathrm{~h}$ incubation with LPS $(1 \mathrm{nM})$ in the absence or presence of MTP $(25 \mu \mathrm{M})$. (C) CCR2 quantification and representative pictures of monocyte flow cytometry analysis after $24 \mathrm{~h}$ incubation with LPS $(1 \mathrm{nM})$ in the absence or presence of MTP $(25 \mu \mathrm{M})$. Values marked with different letters are significantly different $(P<0.05)$.

Figure 2. (A) Mean fluorescence intensity (MFI) of CD68 in M0 macrophages obtained from primary monocytes. CD163 (B), CD64 (C), CD80 (D), CD200r (E), and MR (F) mRNA relative expression in $\mathrm{M} 0, \mathrm{M} 1$, and $\mathrm{M} 2$ macrophages. (G) Production of $\mathrm{NO}_{2}^{-}$in $\mathrm{M} 0$ and $\mathrm{M} 1$ macrophages. MTP $(25 \mu \mathrm{M})$ was added to the medium for $24 \mathrm{~h}$ as indicated. Values marked with different letters are significantly different $(P<0.05)$.

Figure 3. (A) CCR2, (B) CCL2 mRNA relative expression, and (C) migration index in M0, M1, and M2 macrophages. MTP $(25 \mu \mathrm{M})$ and CCL2 $(10 \mathrm{nM})$ were added to the medium for $24 \mathrm{~h}$ as indicated. Values marked with different letters are significantly different $(P<0.01)$.

Figure 4. (A) TNF $\alpha$, (B) IL-1 $\beta$, (C) IL-6 mRNA relative expression; (D) TNF $\alpha$, (E) IL-1 $\beta$ densitometry analysis of immunoblotting detection; (F) TNF $\alpha,(\mathrm{G})$ IL-1 $\beta$, and (H) IL-6 levels in the medium of M0 macrophages after $24 \mathrm{~h}$ incubation with LPS (1 nM) in the absence or presence of MTP $(25 \mu \mathrm{M})$. Values marked with different letters are significantly different $(P$ $<0.05)$. 
FIGURE 1
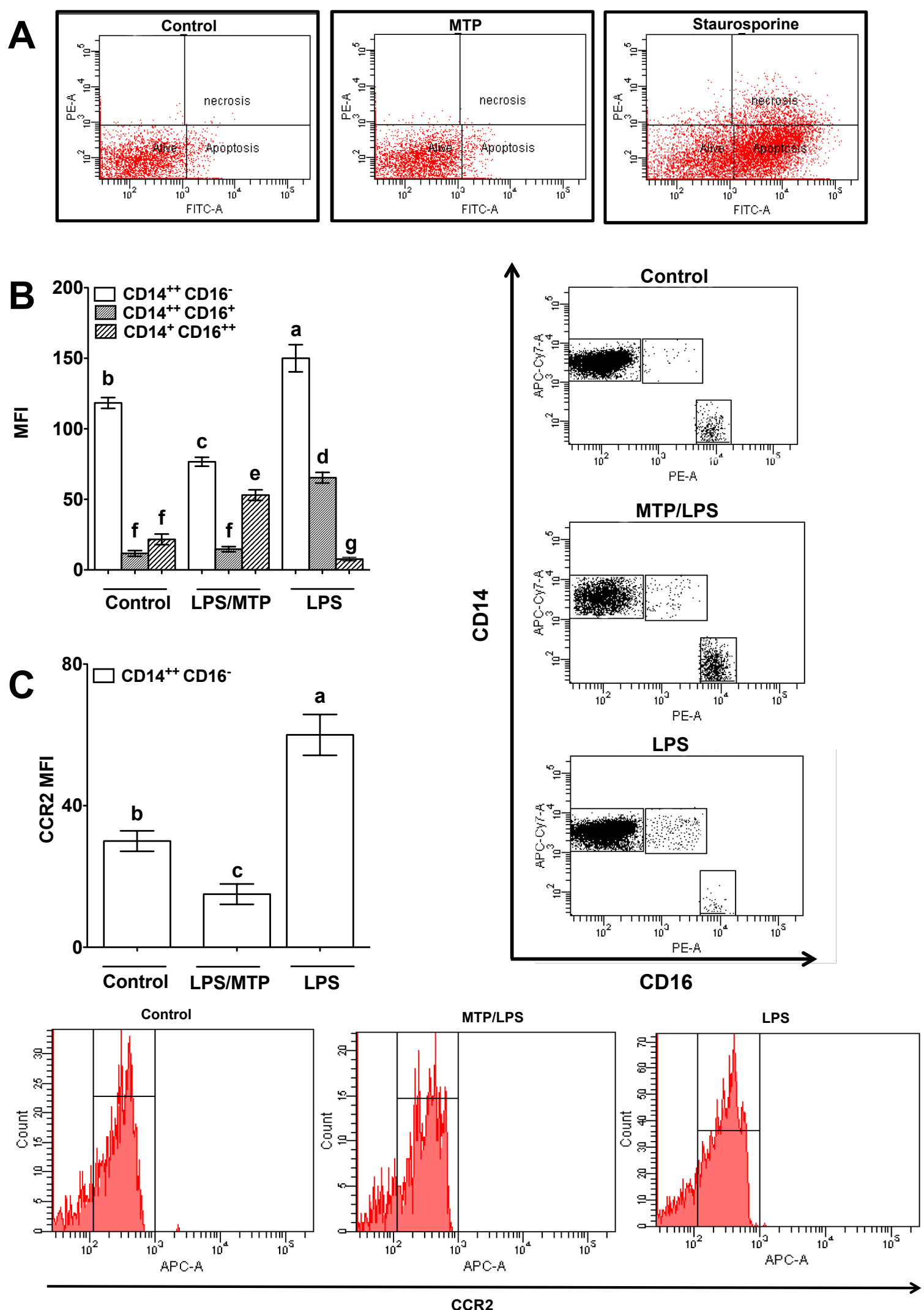

CCR2 
FIGURE 2
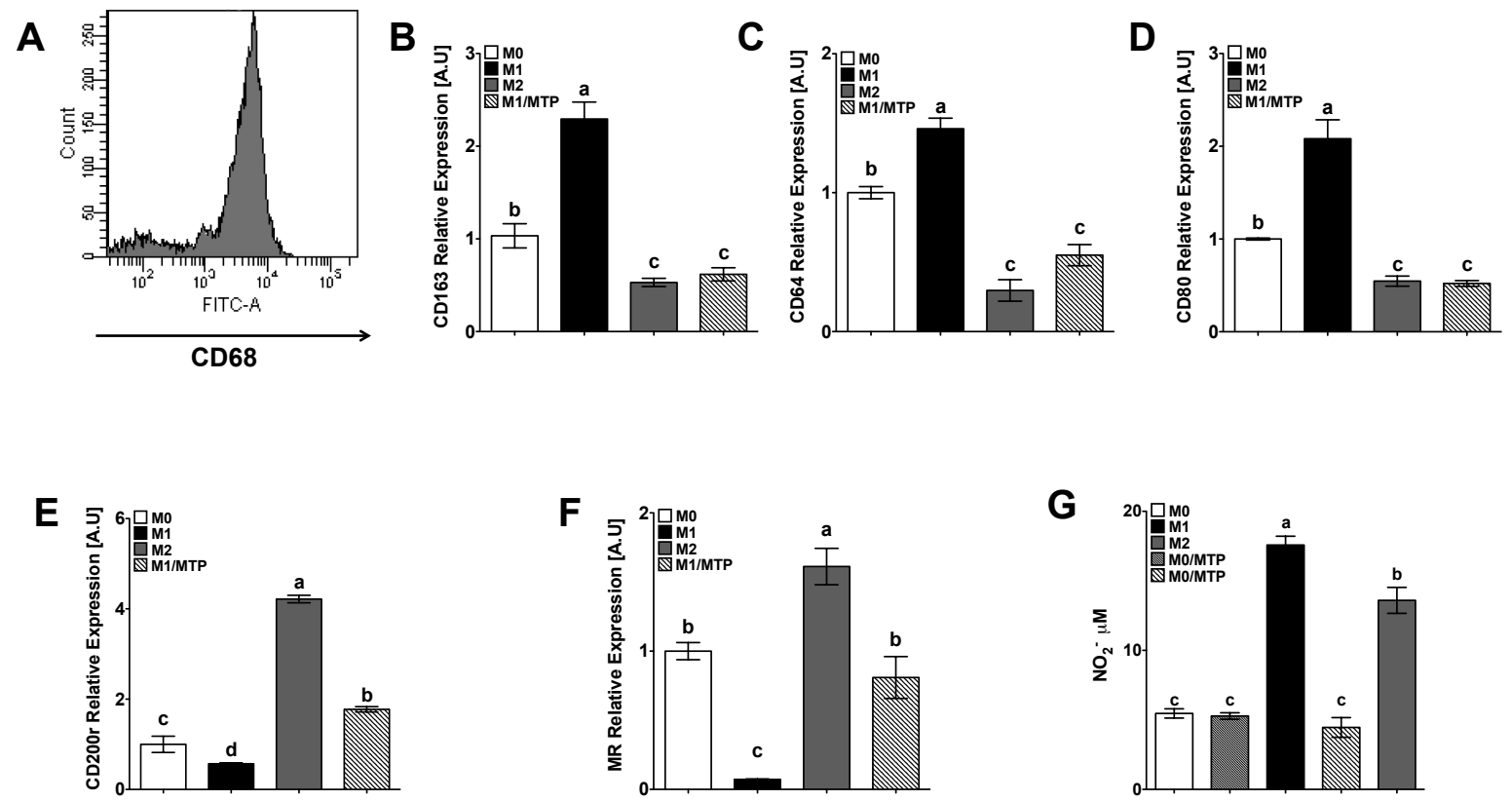
FIGURE 3
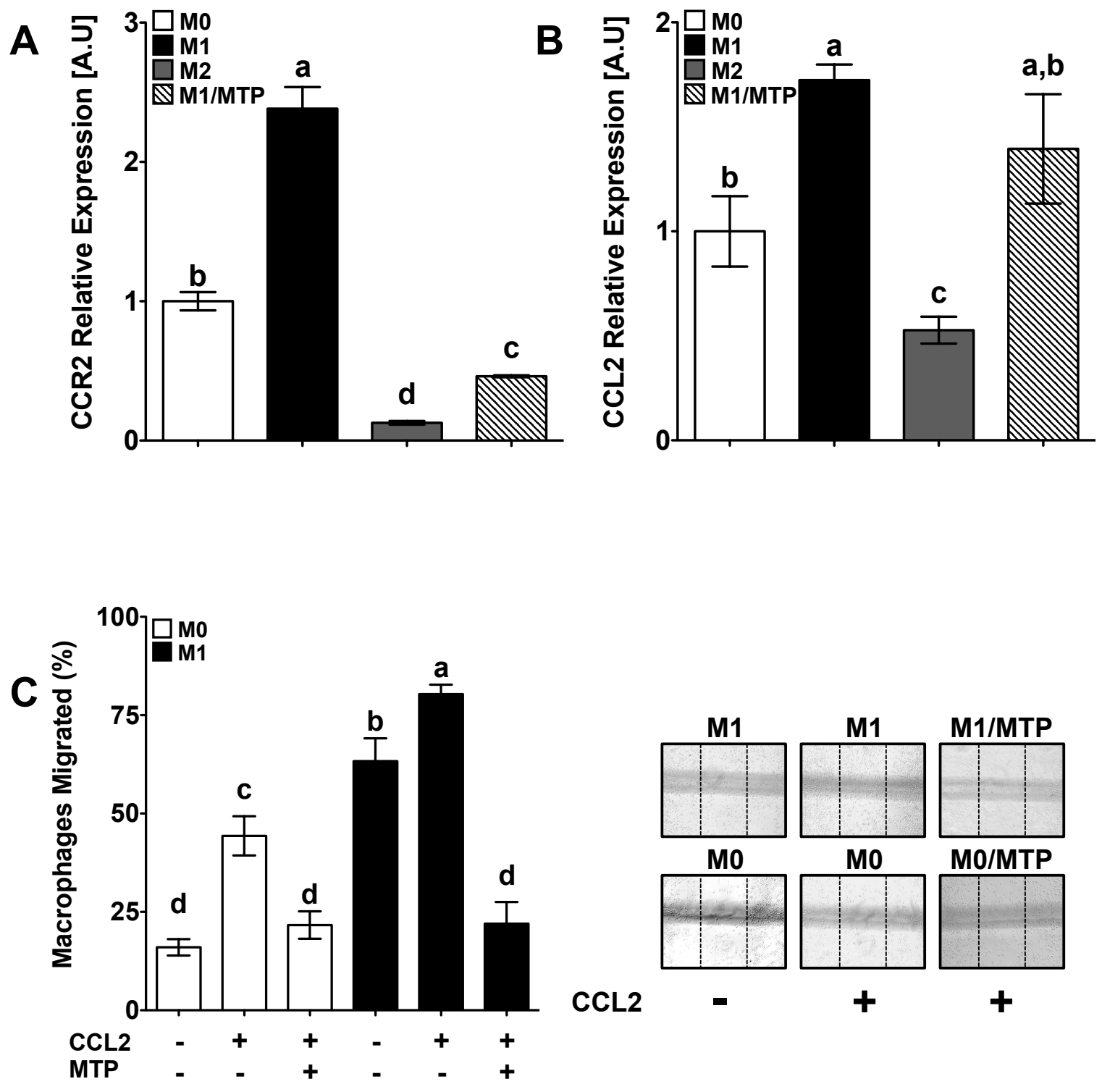
FIGURE 4
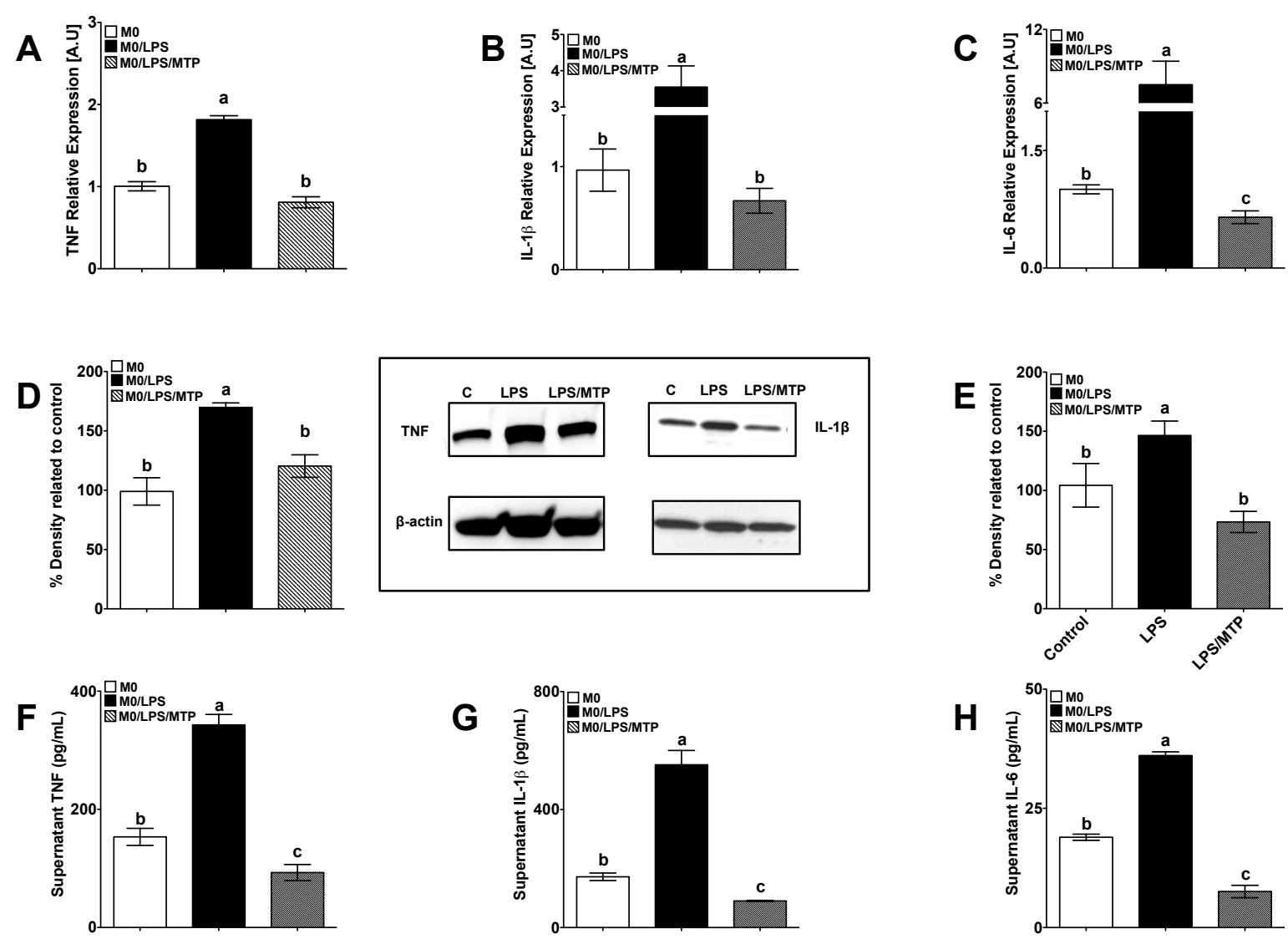